\title{
EFICIENCIA DE USO DE AGUA Y RADIACIÓN EN SOJA BAJO COMPETENCIA CON SORGO DE ALEPO
}

\author{
Pietrobón, M. ${ }^{1}$; ImVinKelRied, H. O. ${ }^{1}$; DellaferReRA, I. M. ${ }^{1,2}$; \\ GARIONE, G. ${ }^{3} \&$ HAIDAR, L. ${ }^{1}$
}

\begin{abstract}
RESUMEN
El objetivo de este trabajo fue evaluar la eficiencia en el uso de agua y radiación (EUA y EUR) de dos variedades de soja creciendo bajo diferentes densidades de plantas, espaciamiento entre surcos y competencia con sorgo de Alepo resistente a glifosato. De forma general, la presencia de malezas redujo la materia seca total, el rendimiento, la EUA y la EUR. El aumento en la densidad y la disminución del espaciamiento entre surcos mejoró la EUA y la EUR e incrementó la materia seca total y el rendimiento. La variedad de mayor grupo de madurez acumuló más materia seca que el de menor grupo de madurez. Sin embargo, ambas variedades presentaron similares rendimientos y EUR.
\end{abstract}

Palabras clave: Glycine max, densidad de plantas, espaciamiento entre surcos, Sorghum halepense.

\begin{abstract}
Use efficiency of water and radiation in soybean under competition with Alepo sorgo.

The objective of this study was to evaluate the use efficiency of water and radiation (WUE and RUE) of two soybeans cultivars growing under different plant densities, row spacing and competition with glyphosate resistant Aleppo sorghum. In general, the presence of weeds reduced total dry matter, yield, WUE and RUE. The increase in density and the de-
\end{abstract}

1.- Cátedra de Cultivos Extensivos. Departamento de Producción Vegetal. Facultad de Ciencias Agrarias, Universidad Nacional del Litoral. Kreder 2805. (3080) Esperanza, provincia de Santa Fe, Argentina. Email: mpietrobon@fca.unl.edu.ar

2.- CONICET.

3.- Alumno cientibecario. Cátedra de Cultivos Extensivos (FCA-UNL).

Manuscrito recibido el 8 de abril de 2019 y aceptado para su publicación el 15 de octubre de 2019.

Pietrobón, M.; Imvinkelried, H.O.; Dellaferrera, I.M.; Garione, G.; Haidar, L. Eficiencia de uso de agua y radiación en soja bajo competencia con sorgo de Alepo. FAVE - Ciencias Agrarias 18 (1): 55-62. CC BY-NC-SA 4.0 (c) (i) (2) 


\section{Pietrobón et al.}

crease in row spacing improved WUE and RUE and increased total dry matter and yield. Soybean cultivar with the highest maturity group accumulated more dry matter than that with the lowest maturity group. However, both cultivars showed similar yields and RUE.

Key words: Glycine max, plant density, row spacing, Sorghum halepense.

\section{INTRODUCCIÓN}

El rendimiento de los cultivos se reduce debido a la competencia por malezas. Para que esto no suceda, la competencia intraespecífica de los cultivos debe reducirse al mínimo y maximizarse el uso de recursos del ambiente $(2,24)$. La competencia por recursos (agua, nutrientes) junto con modificaciones del ambiente bajo el canopeo del cultivo de soja, tales como la cantidad y calidad de luz (14) y temperatura (19) dependientes de la estructura del cultivo (densidad, arreglo espacial, genotipo), generan modificaciones fisiológicas y morfológicas de las malezas que acompañan al cultivo.

El sorgo de Alepo (Sorghum halepense) es una maleza gramínea de amplia difusión y sumamente agresiva con cultivos como soja, maíz o girasol. El principal escollo que interpone esta maleza para su control químico eficaz y económico es su carácter de perennidad a través de rizomas y por su aporte de semillas al suelo (9).

Si bien es sabido que el sorgo de Alepo compite con soja por la luz, agua y nutrientes, reduciendo el rendimiento, la magnitud de la pérdida es dependiente del cultivar y del diseño de distribución espacial del cultivo, el cual ha cambiado notoriamente en los últimos 20 años, sin información al respecto. Resulta interesante considerar un escenario donde el glifosato ya no sea útil para el control de sorgo de Alepo en el cultivo de soja, donde la alternativa de uso de graminicidas selectivos postemergentes podría ir por el mismo camino, para lo cual hay que comprender la importancia de diversificar el manejo como una forma de mitigar el problema.

Los estudios sobre la competitividad del cultivo con las malezas permiten el desarrollo de estrategias para su inclusión en el manejo integrado, dando una ventaja para el agricultor en relación con el manejo de malezas $(1,16)$ al proporcionar otras alternativas, además de la química, para disminuir el efecto negativo de la competencia del sorgo de Alepo sobre el cultivo de soja. El objetivo general del presente trabajo fue evaluar la respuesta a la variación de manejos del cultivo de soja (variedades de diferente grupo de madurez -V-; densidad de plantas -D-; espaciamiento entre surcos -EES-) en la competencia con sorgo de Alepo resistente a glifosato determinando la eficiencia en el uso de recursos agua y radiación y el rendimiento.

\section{MATERIALES Y MÉTODOS}

El ensayo se llevó adelante en la Unidad Experimental de Cultivos Extensivos (FCA-UNL) ubicado en la ciudad de Esperanza (Santa $\mathrm{Fe}$ ), durante la campaña 2017/2018. El diseño experimental fue en 
bloques completos al azar con diseño factorial $2^{4}$-cuatro factores con dos niveles cada uno- y tres repeticiones. Los factores son: a) V: RA 556 -GM V indeterminado- y DM 6.8i -GM VI indeterminado-; b) EES: 52 y $26 \mathrm{~cm}$; c) D: 25 y $35 \mathrm{pl} \mathrm{m}^{-2}$; d) maleza sorgo de Alepo (M): con maleza $\left(10 \mathrm{pl} \mathrm{m}^{-2}\right)$ y sin maleza; haciendo un total de 16 tratamientos.

La siembra del cultivo se llevó adelante con una sembradora experimental de dos surcos. La densidad mínima se estableció en base a resultados de trabajos previos (5). La siembra del sorgo de Alepo se realizó manualmente 10 días posteriores a la siembra del cultivo, en el entresurco, con plantines obtenidos desde semilla.

\section{Materia seca total y rendimiento}

En madurez fisiológica del cultivo se determinó la biomasa total (MS total) (medida en madurez fisiológica -sin hojas-) y el rendimiento (Rto). Se cortaron al ras del suelo las plantas de los dos surcos centrales de cada parcela. Se secaron en estufa de aire forzado a $65{ }^{\circ} \mathrm{C}$ hasta peso constante. Se pesaron para determinar MS total y luego se trillaron para determinar el Rto.

\section{Eficiencia de uso de la radiación}

La eficiencia de uso de la radiación (EUR) se calculó como el cociente entre la MS total y la radiación fotosintéticamente activa (RFA) interceptada (RFAi) acumulada para el ciclo del cultivo -desde emergencia a madurez-. Las mediciones de la RFAi se obtuvieron a través de un radiómetro LICOR modelo LI-250. Se realizaron cinco mediciones por encima de la canopia (I0) y cinco por debajo del nivel de hojas verdes del cultivo (I') entre las 11:30 y 13:00 horas en días soleados, de acuerdo a la metodología propuesta por Gallo y Daughtry (8).
Se obtuvo la RFAi acumulada mediante la multiplicación de la RFA incidente por el $\%$ RI.

\section{Eficiencia de uso del agua}

La eficiencia de uso del agua (EUA) se calculó como el cociente entre la producción de MS total por unidad de agua evapotranspirada por el cultivo (ETc), según fue definido por Pierce y Rice (20). El consumo de agua del cultivo de soja (con y sin maleza) ajustado a condiciones de campo (ETc) se determinó por el método de balance hídrico simplificado $(4,17)$.

\section{Análisis estadístico}

Se utilizó el programa InfoStat, versión 2013 (7). Los datos se evaluaron mediante ANOVA. Las medias se compararon según el test de mínima diferencia significativa (LSD, según sus siglas en inglés) con un nivel de significancia del $5 \%(\mathrm{P} \leq 0,05)$. Cuando existieron interacciones entre los factores principales (V, EES, D y M) se realizó análisis de contrastes.

\section{RESULTADOS Y DISCUSIÓN}

\section{Condición agrometeorológica}

Durante la campaña agrícola las precipitaciones fueron inferiores a lo normal y las temperaturas, tanto máximas como mínimas, levemente superiores a los registros históricos. La precipitación acumulada desde diciembre 2017 hasta abril 2018 fue de 372 mm, un 40,4 \% menos de las Pp históricas. Considerando que un cultivo de soja tardío (siembra de diciembre) necesita en promedio $454 \mathrm{~mm}$ para completar exitosamente su ciclo (6); bajas en el rendimiento logrado pueden asociarse fuertemente a 
este factor climático. Las temperaturas no estuvieron por encima de los rangos de óptimo desarrollo -temperaturas óptimas entre 25 y $35{ }^{\circ} \mathrm{C}$ y, máxima de $40{ }^{\circ} \mathrm{C}$ (13)-. Sin embargo, su principal efecto fue el acortamiento del ciclo de cultivo al acumular más rápidamente los requerimientos térmicos para pasar de una fase de desarrollo a otra.

\section{Materia seca total y rendimiento}

El análisis de las variables MS total y Rto denotaron la interacción de los factores en su respuesta. Para MS total se determinaron interacciones entre $\mathrm{V} * \mathrm{M}(\mathrm{P}=0,0046)$ y $D^{*} \operatorname{EES}(\mathrm{P}=0,0182)$ (Tabla 1$)$. Con relación al Rto se encontró interacción significativa entre todos los factores involucrados $\left(\mathrm{V}^{*} \mathrm{D} * \mathrm{EES} * \mathrm{M}, \mathrm{P}=0,0375\right)$ (Tabla 1).

Tabla 1. Materia seca total (MS total), rendimiento (Rto), eficiencia de uso de la radiación (EUR) y eficiencia de uso del agua (EUA) del cultivo de soja -campaña 2017/2018- según variedad (V; V_GM.Vy V_GM.VI), espaciamiento entre surcos (EES; 52 y $26 \mathrm{~cm}$ ), densidad de plantas $\left(D ; D 1=25 \mathrm{pl} \mathrm{m}^{-2}\right.$ y $D 2=35 \mathrm{pl} \mathrm{m} \mathrm{m}^{-2}$ y maleza (M; con y sin). ns: no significativo; *: $P \leq 0,05$.

\begin{tabular}{|c|c|c|c|c|}
\hline & $\begin{array}{l}\text { MS total } \\
\left(\mathrm{g} \mathrm{m}^{-2}\right)\end{array}$ & $\begin{array}{c}\text { Rto } \\
\left(\mathrm{g} \mathrm{m}^{-2}\right)\end{array}$ & $\begin{array}{c}\text { EUR_MS } \\
\left(\mathrm{g} \mathrm{m}^{-2} \mathrm{MJ}^{-1}\right)\end{array}$ & $\begin{array}{c}\text { EUA_MS } \\
\left(\mathrm{g} \mathrm{m}^{-2} \mathrm{~mm}^{-1}\right)\end{array}$ \\
\hline \multicolumn{5}{|l|}{ V } \\
\hline V_GM.V & 294,4 & 121,3 & $1,04 \mathrm{a}$ & 1,90 \\
\hline V_GM.VI & 353,6 & 129,6 & $1,03 \mathrm{a}$ & 1,29 \\
\hline \multicolumn{5}{|l|}{ EES } \\
\hline 52 & 287,2 & 97,0 & $0,90 \quad b$ & 1,37 \\
\hline 26 & 360,8 & 153,8 & $1,17 \mathrm{a}$ & 1,82 \\
\hline \multicolumn{5}{|l|}{ D } \\
\hline D1 & 264,1 & 116,1 & $0,87 \quad b$ & 1,28 \\
\hline D2 & 383,9 & 134,7 & $1,20 \quad a$ & 1,91 \\
\hline \multicolumn{5}{|l|}{ M } \\
\hline con malezas & 264,7 & 101,6 & $0,83 b$ & $1,30 \mathrm{~b}$ \\
\hline sin malezas & 383,3 & 149,2 & 1,24 a & $1,90 \quad \mathrm{a}$ \\
\hline & \multicolumn{4}{|c|}{ Análisis de varianza } \\
\hline v & * & ns & ns & * \\
\hline EES & * & * & * & * \\
\hline D & * & * & * & * \\
\hline M & * & * & * & * \\
\hline V*EES & Ns & ns & ns & * \\
\hline$V * D$ & Ns & ns & ns & ns \\
\hline$V^{*} \mathbf{M}$ & * & * & ns & ns \\
\hline EES*D & * & ns & ns & * \\
\hline EES*M & Ns & ns & ns & ns \\
\hline$D^{*} M$ & Ns & ns & ns & ns \\
\hline V*EES*D & Ns & ns & ns & ns \\
\hline$V^{*}$ EES*M & Ns & ns & ns & ns \\
\hline$V^{*} D^{*} M$ & Ns & ns & ns & ns \\
\hline EES*D*M & Ns & ns & ns & ns \\
\hline$V^{*} E E S^{*} D^{*} M$ & Ns & * & ns & ns \\
\hline
\end{tabular}


La MS total se incrementó cuando no hubo presencia de malezas, sólo siendo esto significativo en la V_GM.VI con un 71,6\% más de MS. El aumento en la D sólo mejoró la producción de MS total cuando el EES fue mayor, siendo de 86,8 \%. Esto podría asociarse a una mejor distribución de las plantas en la línea de siembra y un mejor aprovechamiento de los recursos disponibles.

El análisis de la interacción $\mathrm{V} * \mathrm{D} * \mathrm{EES}^{*} \mathrm{M}$ permitió determinar que las diferencias estadísticas significativas sólo estuvieron en algunas situaciones (Figura 1). La variedad de GM menor (V_GM.V) sólo logró mayores Rto sin la presencia de malezas a bajas densidades (D1) y EES normales $(52 \mathrm{~cm})$. Por su parte, en la V_GM.VI el Rto se incrementó en todas las situaciones libres de maleza.

La disminución del rendimiento a causa de las malezas es generalmente proporcional a la cantidad de agua, luz y nutrientes usados por estas poblaciones a expensas del cultivo (10). El arreglo espacial puede modificar las relaciones competitivas entre el cultivo y la maleza; cuando esto ocurre, la competencia tiende a aumentar con el menor espaciamiento entre surcos y la distancia entre plantas en la misma hilera (22). Si bien la reducción del EES en muchos casos incrementa la habilidad competitiva del cultivo de soja con las malezas, el resultado puede variar según la especie estudiada, la densidad de la misma y las condiciones ambientales $(22,23)$.

Los valores obtenidos en este trabajo coinciden con los reportes antes mencionados, logrando mayor MS total y Rto con incrementos en la D y con disminuciones del EES y sin malezas. Los mayores GM y los EES más estrechos suelen mejorar la captura de recursos y, por ende, aumentar la MS acumulada y el Rto final obtenido (11). Esto concuerda con Kruk y Satorre (15) quienes explican que el rendimiento

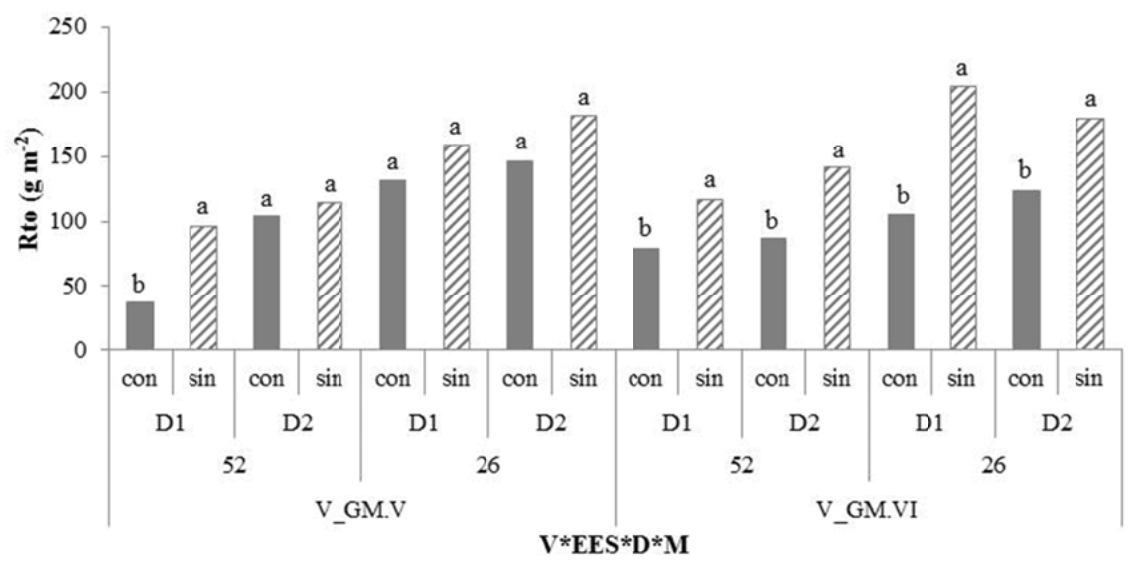

Figura 1. Apertura de la interacción de los factores evaluados sobre el rendimiento (Rto) del cultivo de soja-campaña 2017/2018-segúnvariedad(V;V_GM.VyV_GM.VI), espaciamientoentresurcos (EES; 52 y $26 \mathrm{~cm})$, densidad de plantas $\left(D ; D 1=25 \mathrm{pl} \mathrm{m}^{-2}\right.$ y D2 $\left.=35 \mathrm{plm}^{-2}\right)$ y maleza ( $M$; cony sin). Letras diferentes entre columnas indican diferencias significativas entre cony sin Mdentro de la misma V, EESYD. 
del cultivo tiende a ser mayor a cualquier densidad cuando las plantas se distribuyen regularmente, dado que se minimiza la competencia por los recursos tales como agua, nutrientes y radiación.

\section{Eficiencia de uso de la radiación y del agua}

La presencia de malezas redujo la eficiencia de uso de los recursos radiación y agua (EUR: $\mathrm{P}<0,0001$; EUA: $\mathrm{P}=0,0001$ ) (Tabla 1). Dichas reducciones fueron similares en ambos casos, siendo de 33,1 y 31,6 \% para la EUR y la EUA, respectivamente.

Con relación a la EUR, el estrechamiento de los entresurcos (EES $26 \mathrm{~cm}$ ) y la mayor D de plantas la mejoró. El aumento en la EUR por menor EES fue de 30,0 \% y para la mayor D de $37,9 \%$. La reducción del EES en el cultivo de soja, ha sido sugerida como una práctica de manejo para mejorar la competencia del cultivo sobre la maleza $(18,21)$. Al incrementar la radiación interceptada por el cultivo desde etapas tempranas de su ciclo y, reducir la disponibilidad de este recurso para el crecimiento de la maleza.

Tales efectos han sido verificados en los resultados obtenidos en este trabajo, convalidando la factibilidad de emplear estas herramientas de manejo (D y EES) para disminuir la competencia con las malezas y aumentar la producción y la productividad del cultivo. Los datos logrados coinciden con Imvinkelried (12), quién informa rangos de EUR en MS de 1,05-1,56 $\mathrm{g} \mathrm{m}^{-2} \mathrm{MJ}^{-1}$.

La respuesta en la EUA estuvo influenciada por el efecto de las interacciones entre $\mathrm{V} * \operatorname{EES}(\mathrm{P}=0,0317)$ y $\operatorname{EES} * \mathrm{D}(\mathrm{P}=0,0493)$ (Tabla 1). Con respecto a la interacción $\mathrm{V} *$ EES, la mejora en la EUA se logró estrechando los entresurcos (EES $26 \mathrm{~cm}$ ) sólo en la V_GM.V, logrando un 45,8 \% más de EUA. Mientras que el análisis de EES*D demostró un aumento de $74,2 \%$ en la EUA con disminución del EES sólo en las D menores (es decir, D1 a $26 \mathrm{~cm}$ de EES).

$\mathrm{El}$ agua disponible es, generalmente, el principal factor que limita el crecimiento y Rto de los cultivos en condiciones de secano (3). Los valores obtenidos en este trabajo son consistentes a los reportados por Imvinkelried (12) para la misma área de estudio, quien reportó EUA en MS en el rango de 1,39-2,36 $\mathrm{g} \mathrm{m}^{-2} \mathrm{~mm}^{-1}$, siendo los menores valores en campañas secas y los más altos en campañas húmedas.

\section{CONCLUSIONES}

La presencia de malezas deprimió la MS total, el Rto y la eficiencia de uso de los recursos agua y radiación. Principalmente, el aumento en la D y la disminución del EES mejoró la EUR y EUA. El empleo de una V de GM más largo permitió capturar mayor cantidad de recursos que se tradujeron en una más elevada acumulación de MS, sin presentar diferencias en el Rto final del cultivo. Las precipitaciones menores a las históricas y las temperaturas superiores a los registros normales durante la campaña provocaron un acortamiento del ciclo normal de la soja y pudieron enmascarar cambios en las respuestas de los factores analizados. 


\section{BIBLIOGRAFÍA}

1.- BALBINOT, J. R. 2003. Competitividade de cultivares de arroz irrigado com cultivar simuladora de arroz-vermelho. Pesq. Agropec. Bras. 38 (1): 53-59.

2.- BELLALOUI, N.; BRUNS, H.; ABBAS, H.; MENGISTU, A.; FISHER, D. Y REDDY, K. 2015. Effects of row-type, row-spacing, seeding rate, soil-type, and cultivar differences on soybean seed nutrition under us Mississippi Delta conditions. PLoS ONE. 10: 1-23.

3.- BOYER, J.S. 1982. Plant productivity and environment. Sciencie. 218: 443-448.

4.- CAMUSSI, G.F. Y MARANO, R.P. 2009. Calibración de una sonda de capacitancia para determinación de contenido hídrico en Argiudoles del centro de Santa Fe (pp. 353-358). En: N. Di Leo, S. Montico y G. Nardón (eds.). Avances de la Ingeniería Rural. Ed. UNR. Rosario, Santa Fe, Argentina.

5.- DELLAFERRERA, I.; ARMANDO, M.; PIETROBÓN, M.; IMVINKELRIED, H. Y HERMANN, O.M. 2013. Límites de compensación en el cultivo de soja ante variaciones en la densidad de siembra. Actas II Workshop Internacional de Ecofisiología Vegetal, Mar del Plata.

6.- DELLA MAGGIORA, A.I.; GARDIOL, J.M. E IRIGOYEN, A.I. 2000. Requerimientos hídricos (pp.: 155-171). En: F.H. Andrade y V. Sadras (eds.). Bases para el manejo del maíz, el girasol y la soja. Ed. Médica Panamericana S.A. Buenos Aires. Argentina.

7.- DI RIENZO, J.A.; CASANOVES, F.; BALZARINI, M.; GONZÁLEZ, L.; TABLADA, M. Y ROBLEDO, C. 2013. InfoStat versión 2013. Grupo InfoStat, FCA, Universidad Nacional de Córdoba, Argentina. URL http://www.infostat.com.ar.
8.- GALLO, W.P. Y DAUGHTRY, C.S. 1986. Techniques for measuring intercepted and absorbed photosynthetically active radiation in crop canopies. Agr. J. 78: 752-756.

9.- GHERSA, C.M.; SATORRE, E. Y VAN ESSO, M. 1985a. Seasonal patterns of johnsongrass seed production in different agricultural systems. Israel Journal of Botany. 34 (1): 24-31.

10.- GHERSA, C.M.; LEÓN, R.J.C. Y SORIANO, A. 1985b. Efecto del sorgo de alepo sobre la producción de soja, de maíz y de las malezas presentes en estos cultivos. Rev. Facultad de Agronomía. 6 (3): 123-129.

11.- GIRÓN, P.; JUSTO, C.; LARDONE, A.; BARRACO, M. Y MIRANDA, W. 2014. Comportamiento de cultivares de soja a distintos distanciamientos entre hileras y fechas de siembra. INTA EEA General Villegas. Memoria Técnica 2013-2014: 41-43.

12.- IMVINKELRIED, H.O. 2016. Relación entre la calidad del suelo y variables ecofisiológicas de los cultivos de trigo y soja. Tesis para optar al grado de Doctor en Ciencias Agrarias de la Universidad Nacional del Litoral Ingeniero Agrónomo. Esperanza, Santa Fe. Argentina. $169 \mathrm{p}$.

13.- KANTOLIC, A.G.; GIMÉNEZ, P.I. Y DE LA FUENTE, E.B. 2003. Ciclo ontogénico, dinámica de desarrollo y generación del rendimiento y la calidad de soja (pp.: 167-286). En: E.H.B. Satorre, R.L. Arnold, G.A. Slafer, E.B. de la Fuente, D.J. Miralles, M.E. Otegui y R. Savin (eds.). Producción de granos, bases funcionales para su manejo. FAUBA., Buenos Aires, Argentina.

14.- KASPERBAUER, M.J. 1987. Farred light reflection from green leaves and effects on phytochrome-mediated assimilate partitioning under field conditions. Plant physiology. 85: 350-354. 


\section{Pietrobón et al.}

15.- KRUK, B. Y SATORRE, E.H. 2003. Densidad y arreglo especial del cultivo (pp.: 279316). En: E.H.B. Satorre, R.L. Arnold, G.A. Slafer, E.B. de la Fuente, D.J. Miralles, M.E. Otegui y R. Savin (eds.). Producción de granos, bases funcionales para su manejo. FAUBA., Buenos Aires, Argentina.

16.- LEMERLE, D. 2001. Genetic improvement and agronomy forenhanced wheat competitiveness with weeds. Austr. J. Agric. Res. 52 (5): 527-548.

17.- MARANO, R.P. Y D'ELIA, M. 2005. Estudio del comportamiento de la zona no saturada en parcelas experimentales en el centro de la provincia de Santa Fe. En: Actas XX Congreso Nacional de Agua. Mendoza, Argentina.

18.- NISENSOHN, L. 2006. Características poblacionales de Commelina erecta L. asociadas con su propagación en sistemas cultivados. Tesis presentada para optar al grado de Magíster en Manejo y Conservación de Recursos Naturales. Universidad Nacional de Rosario, Facultad de Ciencias Agrarias. Zavalla, Santa Fe. 92 p.

19.- NORSWORTHY, J.K. Y OLIVEIRA, M. 2007. Tillage and soybean canopy effects on common cocklebur (Xanthium strumarium) emergence. Weed Science. 55: 474-480.

20.- PIERCE, F.J. Y RICE, C.W. 1988. Crop rotation and its impact on efficiency of water and nitrogen use (pp.: 21-42). In: W.L. Hargrove (ed.). Cropping strategies for efficient use of water and nitrogen. ASA-CSSASSSA, Madison, WI.
21.- PROSTKO, E.; CULPEPPER, A.; WEBSTER, T. Y FLANDES, J. 2005. Tropical spiderwort identification and control in Georgia field crops. Tifton, GA: University of Georgia Cooperative Extension Service Bulletin.

22.- PURICELLI, E.C. 1993. Influencia del espaciamiento entre filas y de la competencia de sorgo de Alepo sobre el rendimiento de soja tardía. Pesquisa Agropecuaria Brasileira, Brasilia. 28 (11): 1319-1326.

23.- PURICELLI, E.; FACCINI, D.; ORIOLI, G. Y SABBATINI, M. 2003. Spurred anoda (Anoda cristata) competition in narrow- and wide-row soybean (Glycine max). Weed Technology. 17 (3): 446-451.

24.- WALKER, E.R.; MENGISTU, A.; BELLALOUI, N.; KOGER, C.; ROBERTS, R. Y LARSON, J. 2010. Plant population and row-spacing effects on maturity group III soybean. Agron. J. 102 (3): 821-826. 